\title{
Chronic Obstructive Pulmonary Disease in Patients with HIV: an emerging problem
}

\author{
Andrew T Scourfield, Sarah R Doffman, Robert F Miller
}

Word count excluding Table, Figure legends/key points and references $\sim 2,888$

\section{Introduction}

Clinicians faced with an HIV-infected patient reporting progressive dyspnoea and chronic cough may be familiar with the management of common infectious complications of HIV infection such as Pneumocystis jirovecii pneumonia (PCP) and bacterial pneumonia. However, since the advent of combination antiretroviral therapy (cART) there has been a marked reduction in the incidence of these infectious complications (Heffernan et al, 2005, Grubb et al, 2006). In the HIV-uninfected population, chronic obstructive pulmonary disease (COPD) is a leading cause of mortality and morbidity in the developed world and its economic burden is increasing (Lopez et al, 2006, Mathers and Loncar 2006). There are around 900,000 people currently diagnosed with COPD in the UK and an estimated 2,200,000 people with COPD who remain undiagnosed. This is the equivalent to 13\% of the population of England aged 35 and over (Shahab et al, 2006). With the improved survival seen since cART, COPD is emerging as a significant problem in stable HIV disease and this article describes the epidemiology, pathogenesis, clinical presentation and management of HIV-infected patients with COPD.

Chronic obstructive pulmonary disease is defined as an irreversible airflow limitation, which is usually progressive and associated with an exaggerated chronic inflammatory response to noxious particles in the airways or lung (Vestbo et al, 2013). The chronic airflow limitation that occurs in COPD is caused by a combination of small airways disease (obstructive bronchiolitis) and parenchymal destruction (emphysema). The disease course is characterised by frequent exacerbations of symptoms, which have also been shown to contribute to an accelerated decline in lung function.

Spirometry is the most widely available and reproducible test of lung function and can demonstrate evidence of airflow obstruction required to diagnose COPD. Forced expiratory volume in 1 second (FEV1) and forced vital capacity (FVC) are measured and a post-bronchodilator FEV1/FVC ratio of $<0.70$ confirms airflow obstruction. In those with evidence of irreversible airways obstruction with an FEV1/FVC of $<0.70$, the predicted FEV1 can be used to give an estimation of disease severity (Vestbo et al, 2013). With progressive destruction of lung parenchyma, gas exchange, as measured by diffusion capacity of the lung for carbon monoxide (also known as transfer factor), is impaired and is a manifestation of emphysema. Small airways collapse can be measured by assessing the speed of air leaving the lung during the middle portion of a forced expiration, called forced expiratory flow (FEF) 25-75\%. Gas trapping on expiration, can be measured by increased residual volumes on lung function testing. 


\section{Epidemiology}

Respiratory symptoms such as cough and dyspnoea were commonly reported among HIV-infected patients early in the epidemic. The high prevalence was attributed to pulmonary infection, HIV related inflammation and high rates of cigarette smoking. Early studies of pulmonary function revealed transfer factor (TLCO) declined with progression of HIV disease in keeping with this hypothesis. In the early 1990's, precART, a case series (Diaz et al, 1992) reported emphysematous changes on CT imaging and abnormal lung function testing in four individuals (aged $<55$ years) in the absence of pulmonary infection with limited smoking history. The same group performed a subsequent case controlled study of 114 HIV-infected individuals without pulmonary infections matched for age and smoking with $44 \mathrm{HIV}$-uninfected controls. This showed the presence of emphysema in 15\% compared with $2 \%$ in the non-HIV infected group (Diaz et al, 2000). Abnormalities in diffusion capacity have also been noted in the cART era. A recent cross-sectional analysis of $300 \mathrm{HIV}$-infected men and $289 \mathrm{HIV}$-uninfected men enrolled from 2009 to 2011 in the Lung HIV Study at several study sites in the US showed that, despite most participants having normal airflow, there was a significant reduction in TLCO in HIV infected compared with HIV-uninfected men (mean predicted $69 \%$ vs $76 \%$ ). A moderate to severe reduction of TLCO ( $\leq 60 \%)$ was observed in $30 \%$ of HIV-infected compared with $18 \%$ of HIV-uninfected men, despite the fact that $89 \%$ of those with HIV were on cART. A reduced TLCO was significantly associated with being $\mathrm{HIV}$ infected and with lower CD4 cell count ( $<200$ cells/ $\mu \mathrm{l})$, after adjusting for smoking and other confounders. Respiratory symptoms of cough, sputum and dyspnea were more prevalent in HIV-infected patients, particularly those with abnormal pulmonary function compared with HIV-uninfected patients (Crothers et al, 2013).

In the post-cART era, a prospective observational study from the US of 1,014 HIVinfected and 713 HIV-uninfected men from the Veterans Aging Cohort Study (VACS) assessed prevalence of COPD as determined by International Classification of Diseases, ninth revision (ICD-9). After adjusting for age, race/ethnicity, pack-years of cigarette smoking, IVDU, and alcohol misuse, HIV infection was an independent risk factor, with COPD 50-60\% more likely in those with HIV (Crothers et al, 2006). This finding was confirmed in a larger follow up study of the same cohort (Crothers et al, 2011). There are now several studies from North America and one from Europe have assessed the prevalence and risk factors obstructive airways disease in HIV-infected individuals. A comprehensive list of these studies and the key findings are shown in table 1. Data from Brighton, UK has shown $15 \%$ prevalence of airflow obstruction (FEV1:FVC $<0.7$ ) postbronchodilator in 133 consecutive patients recruited from an HIV outpatient clinic (Dickson et al, 2013).

\section{Pathogenesis}

The respiratory tract serves as an interface between the host and the environment; pulmonary immune defences encompass both innate and acquired responses. Pathogens entering the respiratory tract are phagocytosed by alveolar macrophages, the main component of the innate immune system in the lung. Alveolar macrophages are generally immunosuppressive to prevent an inflammatory response. If the innate host defence is impaired, persistence of the antigen stimulates specific acquired immune responses. This occurs in the lymphoid tissue of the lung and results in 
production of antigen specific T and B cells; under the control of local chemokine production, these migrate to the sites of the initial challenge. Studies have demonstrated the accumulation of CD8+ T-cells in the lungs of patients with severe COPD; HIV positive smokers have also been shown to have a higher percentage of CD8+ T cells detectable on bronchoalveolar lavage. HIV infection causes activation of alveolar macrophages, a reduction in CD4 T-cell count, increased number and percentage of CD8 T-cells and an increased CD4:CD8 ratio in the alveolar space with generalized CD4 and CD8 T-cell activation (Beck J, 2013, Twigg $\mathrm{H}$ and Knox S, 2013). This leads to a cycle of pulmonary inflammation and damage.

\section{Role of Pneumocystis jirovecii colonisation}

The lung microbiome is a rapidly expanding area of research and studies have shown wide variability in the microbiological population in the lungs of HIV-infected as compared with uninfected individuals (Ireland et al, 2012, Twigg et al, 2010 and 2011).

Pneumocystis jirovecii is a well-recognised cause of pneumonia (PCP) in those with HIV infection. In HIV-uninfected individuals, colonisation with $P$. jirovecii is a risk factor for COPD severity, independent of smoking history or corticosteroid use. In HIV-infected patients the frequency of $P$. jirovecii colonisation has been reported as $40-58 \%$. While those with lower CD4 counts appear to have increased rates of colonisation, other studies have suggested that this still occurs in those on antiretroviral therapy, with higher CD4 counts and in those taking PCP prophylaxis (Morris and Norris, 2012).

A study of HIV-infected individuals showed worse airflow obstruction in those colonized with $P$. jirovecii, even adjusting for smoking history. Animal studies also suggest colonisation with pneumocystis may play a role in the development of COPD. One study in primates revealed macaques infected with simian immunodeficiency virus (SIV) developed pneumocystis colonisation and these animals had higher rates of airflow obstruction and increased emphysematous changes. Immunocompetent mice colonised by pneumocystis and exposed to cigarette smoke were more likely to develop emphysema than non-colonized mice or those not exposed to cigarette smoke, implying these two factors are synergistic (Morris and Norris, 2012).

The inflammatory response to pneumocystis in the lung is similar to that seen in COPD, even when $P$. jirovecii is detected at low levels, as occurs in colonisation. These changes include increased numbers of CD8 lymphocytes, neutrophils and macrophages. It remains to be determined if colonisation with pneumocystis in those with COPD is a cause of ongoing inflammation or a marker of structural lung damage and use of corticosteroids (Morris and Norris, 2012).

\section{Clinical presentation}

A diagnosis of COPD should be considered in any HIV-infected patient who reports dyspnoea, chronic cough or sputum production, particularly in those with a history of exposure to a risk factor such as cigarette smoke, occupational exposure to dust and chemicals, irrespective of the CD4 count and whether or not the patient is taking cART. 
Chronic cough is often the first symptom of COPD to develop, the cough may be dry, but commonly patients with COPD produce small amounts of sputum. Large volume sputum production should raise the suspicion of underlying bronchiectasis.

Dyspnoea is another common symptom in individuals with COPD and frequently encountered in HIV-infected persons with a wide differential diagnosis. In the early stages dyspnoea may be reported on exertion but as lung function deteriorates increasing breathlessness may occur at rest and cause significant disability and anxiety. PCP should always be considered in those who are not receiving cART and who have low CD4 counts $(<250$ cells $/ \mu \mathrm{l})$. Wheezing and chest tightness are nonspecific symptoms that may also be present, usually after exertion, however their absence does not exclude COPD.

The differential diagnosis for HIV infected patients with chronic cough and dyspnoea is shown in Table 2.

It is important to assess for other features of severe COPD such as ankle swelling which may suggest the development of right heart failure from cor pulmonale. Careful attention should also be paid to detecting anxiety or depression, which are associated with worse outcomes and more frequent in individuals with HIV (Rabkin, 2008). Weight loss and anorexia may represent advanced COPD or suggest an alternative pathology.

\section{Imaging}

\section{Chest radiograph}

In those with chronic cough and shortness of breath chest radiography should be a first line investigation. It is useful to exclude other causes such as malignancy or tuberculosis, and during exacerbations to exclude complications of COPD such as pneumonia and pneumothorax. In COPD the chest radiograph may show hyperexpanded lung fields ( $>7$ th anterior rib intersecting the diaphragm at the midclavicular line), flattening of the hemidiaphragms, and if bullous lung disease is present this may be seen as a hyperlucency in lung fields (Figure 1). 


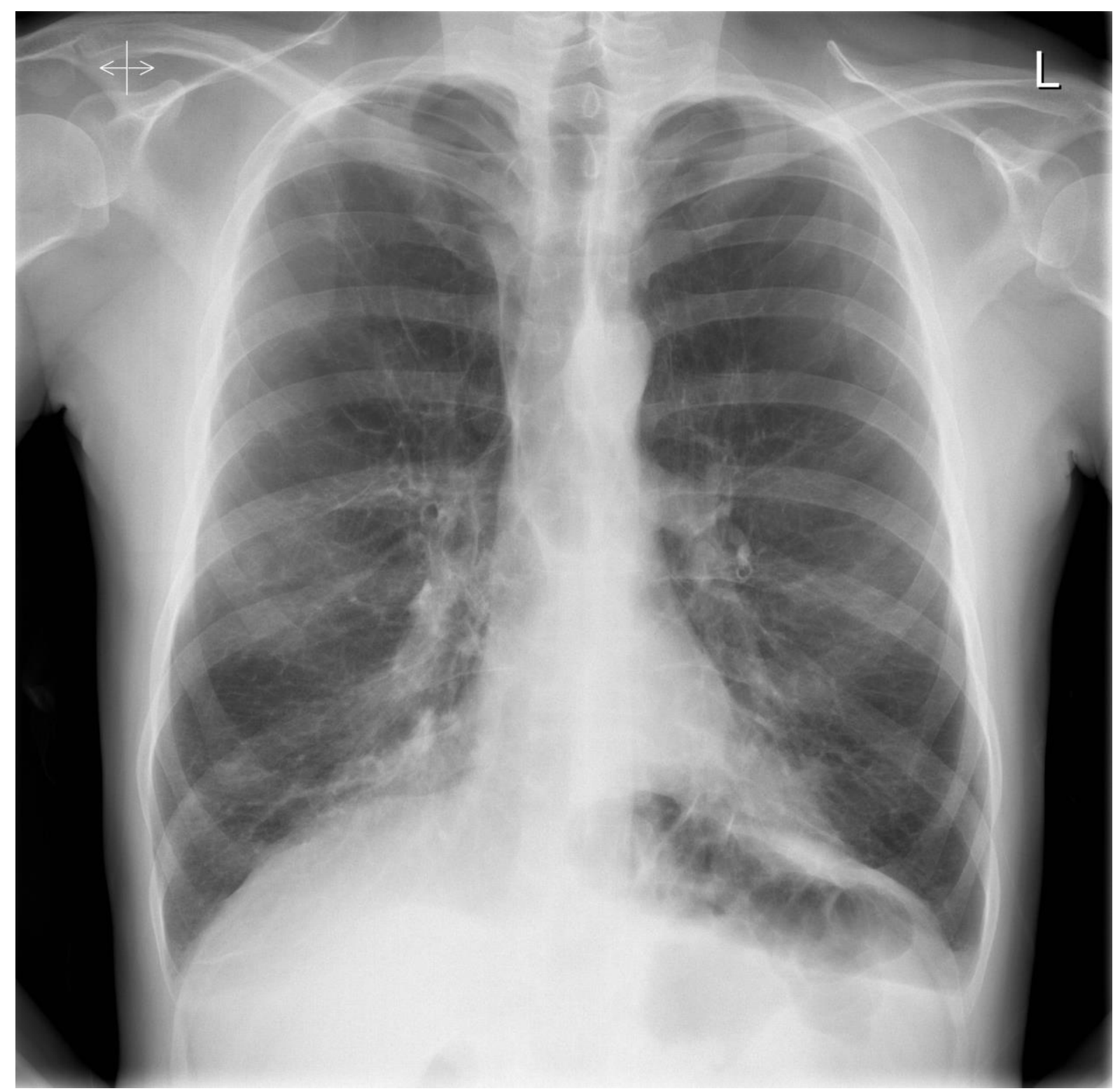

Figure 1. Radiograph of 50-year-old male ex-smoker, diagnosed with HIV 1998.

Recurrent pneumonia. Started cART 2006, current CD4 300 cells/ $\mu \mathrm{l}$, VL $<50$ copies/ml

\section{Computed tomography (CT) chest}

In the late 1980s a high prevalence of bullous lung disease was noted on CT scans of HIV-infected individuals and occurring at a younger age (mean 37 years) compared to other immunocompromised individuals (Kuhlman et al, 1989). Features of COPD seen on chest CT include: prominent small airways, emphysema which can be centriacinar, panacinar and para-septal and mosaic attenuation due to gas trapping from obstructive bronchiolitis. Expiratory phase high resolution CT scan can show evidence of airtrapping, which in some patients may be the only sign of an early-stage small airways disease in an otherwise normal lung. More severe disease is characterized by the development of thin walled bullae. (Figure 2). 


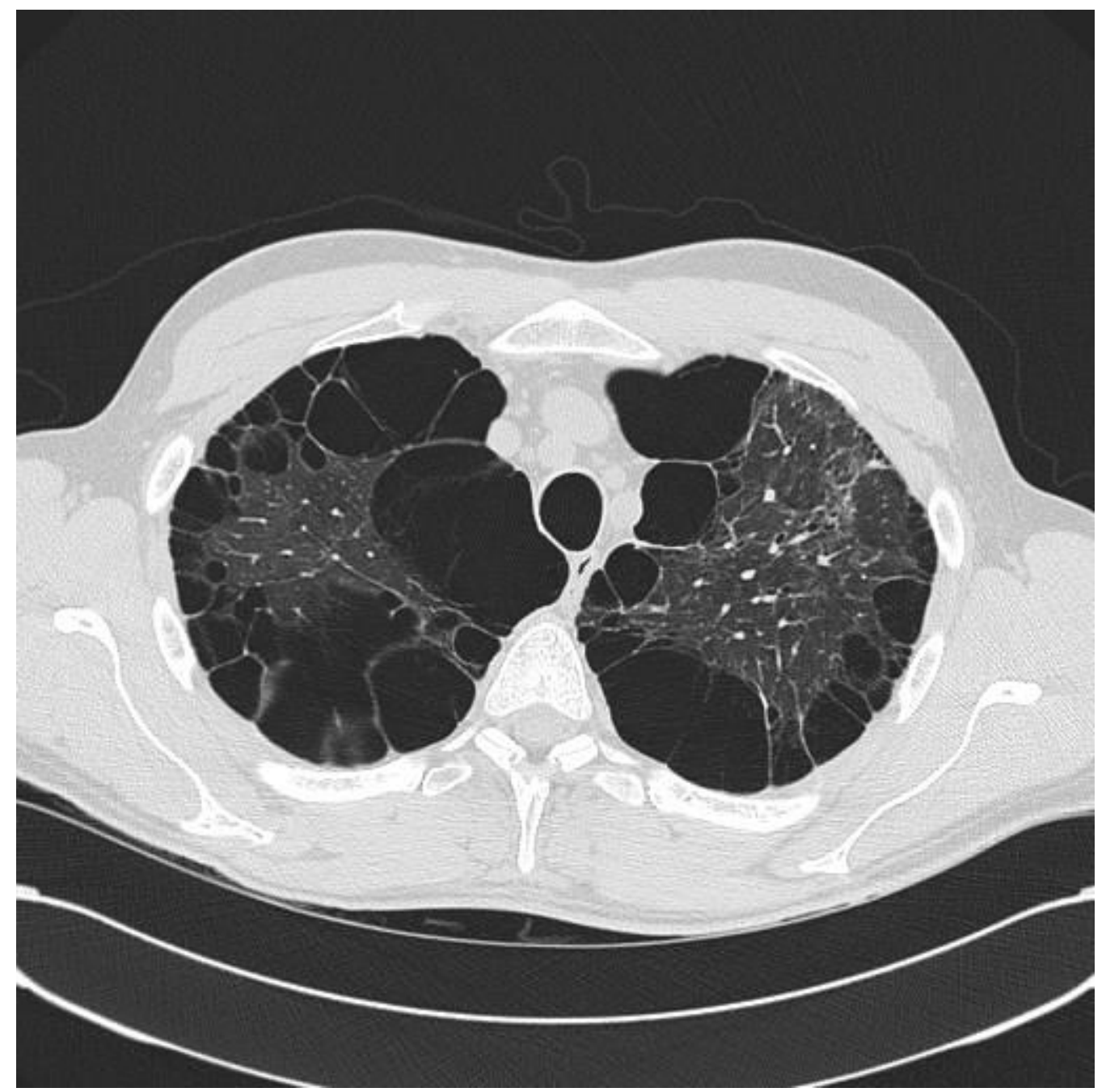

Figure 2. CT chest of 44 year old male, current smoker. HIV diagnosed 2004 with PCP infection. Started cART 2009 poor compliance, CD4 170 cells/ $\mu$ l, VL >100,000 copies/ml

\section{Important considerations when managing HIV-infected patients with COPD}

\section{Pharmacotherapy and drug-drug interactions}

In the absence of data specific to the HIV population pharmacotherapy for COPD is in line with guidelines for the HIV-uninfected population. Inhaled bronchodilators (B2 agonists and anticholinergics) are the preferred initial therapy with inhaled corticosteroids reserved for those with severe disease.

There are several important interactions for prescribers to be aware:

- Inhaled corticosteroids. Multiple case reports describe iatrogenic Cushing's syndrome in individuals on ritonavir-boosted protease inhibitor antiretroviral regimen treated with fluticasone (a component of Seretide ${ }^{\circledR}$ ) due to reduced steroid metabolism (Foisy et al, 2008). Ritonavir is a potent inhibitor of the hepatic CYP3A4 isoenzyme. Other inhaled steroids metabolised by this route include budesonide and mometasone; beclomethasone is not and can be safely administered with ritonavir containing antiretroviral therapy. Of note, the new pharmacoenhancer cobicistat, currently only available as part of a fixed dose 
combination called Stribild ${ }^{\circledR}$, is also a potent CYP3A4 inhibitor and therefore should not be administered with inhaled steroids other than beclomethasone. Inhaled corticosteroids should be used with caution in HIV-infected individuals as these are known to increase risk of bacterial pneumonia (Calverley et al, 2007) and this risk remains elevated despite the use of cART (Heffernan et al, 2005).

- Long acting beta-agonists. Co-administration of salmeterol (also contained in Seretide ${ }^{\circledR}$ ) with ritonavir is not recommended due to increased salmeterol concentrations that may result in QT prolongation, palpitations and sinus tachycardia (Norvir SPC 2012).

- Short and long acting anticholinergics are thought to be safe with cART although this interaction has not been studied.

- Oral prednisolone is frequently used in short courses to treat COPD exacerbations. A study of co-administration of prednisolone $20 \mathrm{mg}$ once daily with ritonavir 200mg twice daily (a higher dose than used in clinical practice) showed an increased area under the curve of the active metabolite prednisolone by $37 \%$ and $28 \%$ after 4 and 14 days of ritonavir, respectively (Norvir SPC 2012). This is unlikely to be of clinical significance in short courses but monitoring for therapeutic and adverse effects is advised with prolonged courses.

\section{Role of antiretroviral therapy}

Due to the early onset of COPD in HIV, surrogate markers have been investigated; the most studied factor is the impact of HIV viral load. Poorly controlled HIV has been associated with worse lung function; the afore-mentioned VACS cohort study described earlier showed COPD was less likely in those with suppressed HIV RNA levels $(<400$ copies $/ \mathrm{ml}$ ) and those on cART at baseline (Crothers et al, 2011). Another study of 1077 former injection drug users demonstrated that airway obstruction was associated with HIV viral loads $>200,000$ copies/ml. In a longitudinal study follow up of this cohort over median 2.75 years, high HIV viral load ( $>75,000$ copies/ml) and low CD4 count $(<100$ cells/ $\mu \mathrm{l}$ ) were associated with reduced FEV1 and FVC (Drummond et al, 2013).

With the initiation of cART there is a trend to normalisation of pulmonary immune function. Despite this, two studies have suggested worse airflow obstruction in patients on cART (George et al, 2009, Gingo et al, 2010). Possible explanations for this observation might be the timing of cART in different populations or immune response to subclinical infections.

\section{Similarities to managing COPD in the general population}

\section{Smoking cessation}

The prevalence of cigarette smoking among HIV-infected individuals ranges from 44$86 \%$ depending on the population studied. Smoking cessation in the non-HIV 
population has been shown to delay decline in lung function (Fletcher and Peto, 1977) and should be the main focus of any treatment strategy. Most smoking cessation trials use motivational interviewing with or without nicotine replacement therapy. The success reported for smoking cessation interventions in individuals with HIV has shown variable results. A study of 474 HIV positive smokers, randomised to usual care or counselling via mobile phone intervention demonstrated significantly greater abstinence up to 3 months; however, the effect diminished after 6 and 12 months (Gritz et al, 2013). Another randomised study of 209 HIV positive smokers to nicotine replacement combined with counselling, self-help or internet-based treatment all arms showed comparable results (Humfleet et al, 2013). The largest study of varenicline compared it to nicotine replacement therapy, both with telephone counselling in 228 HIV positive smokers. This showed promising results with biochemically confirmed abstinence rates at three months of $25.6 \%$ vs $11.8 \%$ respectively. The adverse event profile was similar to the general population and there was no association of adverse events with cART use (Ferketich et al, 2013).

\section{Pulmonary rehabilitation}

The combination of COPD and HIV is associated with higher rate of self-reported physical disability and decreased physical functioning (Oursler et al, 2011). In HIVuninfected individuals with COPD, pulmonary rehabilitation significantly improves physical functioning. Systemic effects of inflammation from HIV can contribute to reduction in muscle mass and exercise capacity. Aerobic exercise appears to be safe and may be beneficial for HIV infected adults but the optimal exercise regime for those with HIV and COPD is yet to be determined (Nixon et al, 2010).

\section{Conclusions and future perspectives}

COPD is a major cause of morbidity and mortality in the UK. With the ageing HIV population and due to the insidious onset of symptoms, physicians managing those with HIV should enquire routinely about smoking habits and pay particular attention to eliciting symptoms of COPD such as cough, dyspnoea, sputum production and wheezing.

Even after controlling for known risk factors COPD appears to be more prevalent in individuals with HIV. This may be related to chronic inflammatory changes that occur as well as immune response to subclinical microorganisms colonising the respiratory tract.

The prevalence of cigarette smoking in the HIV-population is extremely high and the key focus of any treatment strategy should be smoking cessation. Pharmacotherapy is in line with national guidance for management of COPD, but there are significant drugdrug interactions which patients, General Practioners and Specialists need to be aware of (particularly those taking CYP3A4 inhibitors, such as ritonavir and cobicistat).

Complex patients may benefit from early involvement of a respiratory specialist with an interest in HIV.

Novel treatment strategies may look at using anti-inflammatory agents and assessing response to eradication of PCP in those who are colonised. Antiretroviral therapy is important to prevent pulmonary opportunistic infections and to reverse systemic and intrapulmonary inflammation. Given the trend for earlier initiation of cART it will be 
interesting to see how this will impact on the incidence of COPD in HIV-infected individuals.

\section{Key points}

- Physicians caring for HIV infected patients should be alert for COPD

- Even after controlling for cigarette smoking COPD appears to be more prevalent among HIV-infected persons than among the general population

- Treatment is as for general population - i.e. smoking cessation and pharmacologic interventions

- Clinically significant drug-drug interactions www.hiv-druginteractions.org

Conflict of interest: Professor Rob Miller is Editor in Chief of British Journal of Hospital Medicine. He took no part in the peer review process nor in the decision to accept it for publication

\section{$\underline{\text { References }}$}

Beck J. (2013) Clinics in chest medicine, HIV and Respiratory Disease

Calverley PM, Anderson JA, Celli B, et al. (2007) Salmeterol and fluticasone proprionate and survival in chronic obstructive pulmonary disease. $N$ Engl J Med;356(8):775-89

Crothers K, Butt AA, Gibert CL, et al. (2006) Increased COPD among HIV-positive compared to HIV-negative veterans. Chest;130(5)1326-33

Crothers, K, Huang L, Goulet JL, et al. (2011) HIV infection and risk for incident pulmonary diseases in the combination antiretroviral therapy era. Am J Respir Crit Care Med; 183(3):388-95

Crothers K, McGinnis K, Kleerup E, et al. (2013) HIV Infection Is Associated With Reduced Pulmonary Diffusing Capacity. J Acquir Immune Defic Syndr. Nov 1;64(3):271-8

Cui Q, Carruthers S, McIvor A, et al. (2010) Effect of smoking on lung function, respiratory symptoms and respiratory diseases amongst HIV-positive subjects: a crosssectional study. AIDS Res Ther. Mar 19;7:6

Diaz PT, Clanton TL, Pacht ER. (1992) Emphysema-like pulmonary disease associated with human immunodeficiency virus infection. Ann Intern Med; 116(2):124-8

Diaz PT, King MA, Pacht ER, et al. (2000) Increased susceptibility to pulmonary emphysema among HIV-seropositive smokers. Ann Intern Med. Mar 7;132(5):369-72. 
Dickson N, Hollington R, Malbon R, et al. (2013) Prevalence of chronic obstructive pulmonary disease in an HIV infected population. HIV Med. 14 (suppl 2):12-77 (abstr P95)

Drummond MB, Kirk GD, Astemborski J, et al. (2012) Association between obstructive lung disease and markers of HIV infection in a high-risk cohort. Thorax. 67(4):309-14

Drummond MB, Merlo CA, Astemborski J, et al. (2013) The effect of HIV infection on longitudinal lung function decline among IDUs: a prospective cohort. AIDS. 15;27(8):1303-11

Ferketich AK, Diaz P, Browning KK, et al. (2013) Safety of varenicline among smokers enrolled in the lung HIV study. Nicotine Tob Res. 15(1):247-54

Fletcher C, Peto R. (1977) The natural history of chronic airflow obstruction. BMJ;i:1645-8

Foisy MM, Yakiwchuk EM, Chiu I, et al. (2008) Adrenal suppression and Cushing's syndrome secondary to an interaction between ritonavir and fluticasone: a review of the literature. HIV Med. 9(6): 389-96

George MP, Kannass M, Huang L, et al. (2009) Respiratory symptoms and airway obstruction in HIV-infected subjects in the HAART era. PLoS One. 21;4(7):e6328

Gingo MR, George MP, Kessinger CJ, et al. (2010) Pulmonary function abnormalities in HIV-infected patients during the current antiretroviral therapy era. Am J Respir Crit Care Med. Sep 15;182(6):790-6

Gritz ER, Danysh HE, Fletcher FE, et al. (2013) Long-term outcomes of a cell phonedelivered intervention for smokers living with HIV/AIDS Clin Infect Dis. 57(4):608-15.

Grubb JR, Moorman AC, Baker RK, et al. (2006) The changing spectrum of pulmonary disease in patients with HIV infection on antiretroviral therapy. AIDS. 12;20(8):1095107

Heffernan RT, Barrett NL, Gallagher KM et al. (2005) Declining incidence of invasive Streptococcus pneumoniae infections among persons with AIDS in an era of highly active antiretroviral therapy, 1995-2000.J Infect Dis. 15;191(12):2038-45

Hirani A, Cavallazzi R, Vasu T, et al. (2011) Prevalence of obstructive lung disease in HIV population: a cross sectional study. Respir Med.105(11):1655-61

Humfleet GL, Hall SM, Delucchi KL, et al. (2013) A randomized clinical trial of smoking cessation treatments provided in HIV clinical care settings. Nicotine Tob Res. 15(8):1436-45

Ireland AW, Ghedin E, Pop M, et al. (2012) Comparison of the respiratory microbiome in HIV-infected and HIV-uninfected individuals. Am J Resp Crit Care Med;185(Meeting abstracts:A4045 
Kristoffersen US, Lebech AM, Mortensen J, et al. (2012) Changes in lung function of HIVinfected patients: a 4.5-year follow-up study. Clin Physiol Funct Imaging; 32(4):288-95

Kuhlman JE, Knowles MC, Fishman EK, et al. (1989) Premature bullous pulmonary damage in AIDS: CT diagnosis. Radiology;173:23-6

Lopez AD, Shibuya K, Rao C, et al. (2006) Chronic obstructive pulmonary disease: current burden and future projections. Eur Respir J;27:397-412.

Mathers CD, Loncar D. (2006) Projections of global mortality and burden of disease from 2002 to 2030. PLoS Med;3:e442

Morris A, Norris K. (2012) Colonization by Pneumocystis jirovecii and its role in disease. Clin Microbiol Rev.

Nixon S, O'Brien K, Glazier RH, et al. (2010) Aerobic exercise interventions for adults living with HIV/AIDS. Cochrane Database Syst Rev;(8):CD001796

Norvir Summary of Product Characteristics, (2012) Abbott Laboratories Ltd

Oursler KK, Goulet JL, Crystal S, et al. (2011) Association of age and comorbidity with physical function in HIV infected and uninfected patients: results from the Veterans Aging Cohort Study. AIDS Patient Care STDS; 25(1):13-20

Rabkin JG. (2008) HIV and Depression: review and update. Curr HIV/AIDS Rep. 5(4):163-71

Shahab L, Jarvis MJ, Britton J, et al. (2006) Prevalence, diagnosis and relation to tobacco dependence of chronic obstructive pulmonary disease in a nationally representative population sample. Thorax, 61(12):1043-7

Twigg HL, Nelson D, Dong Q, et al. (2010) Analysis of the respiratory microbiome using bronchoalveolar lavage from HIV -infected and uninfected subjects. Am J Respir Crit Care Med;181(Meeting abstracts):A5629

Twigg HL, Nelson D, Dong Q, et al. (2011) Analysis of the respiratory microbiome using bronchoalveolar lavage from HIV-infected and uninfected subjects. Am J Respir Crit Care Med;183(Meeting abstracts):A6257

Twigg H, Knox S. (2013) Clinics in chest medicine, HIV and Respiratory Disease

Vestbo J, Hurd S, Agusti A, et al. (2013) Global Strategy for the Diagnosis, Management, and Prevention of Chronic Obstructive Pulmonary Disease. American Journal of Respiratory and Critical Care Medicine, Vol. 187, No. 4, pp. 347-365 